Table 2. Numbers and combinations of autoantibodies identified in the 96 SSc patients.

\begin{tabular}{|c|c|c|c|c|c|c|c|c|c|c|}
\hline & Topo-I & CENP & RNAP III & Fibrillarin & NOR90 & $\mathrm{Th} / \mathrm{To}$ & $\mathrm{Pm} / \mathrm{Scl}$ & $\mathrm{Ku}$ & PDGFR & Ro52 \\
\hline Topo-I & 22 & 1 & 7 & 0 & 1 & 5 & 9 & 5 & 0 & 7 \\
\hline CENP & & 12 & 2 & 0 & 2 & 2 & 2 & 2 & 0 & 11 \\
\hline RNAPIII & & & 4 & 0 & 1 & 2 & 1 & 3 & 0 & 7 \\
\hline Fibrillarin & & & & 0 & 0 & 0 & 0 & 0 & 0 & 0 \\
\hline NOR90 & & & & & 0 & 1 & 1 & 0 & 0 & 2 \\
\hline $\mathrm{Th} / \mathrm{To}$ & & & & & & 0 & 4 & 2 & 0 & 2 \\
\hline $\mathrm{Pm} / \mathrm{Scl}$ & & & & & & & 4 & 3 & 0 & 6 \\
\hline $\mathrm{Ku}$ & & & & & & & & 1 & 0 & 2 \\
\hline PDGFR & & & & & & & & & 0 & 0 \\
\hline Ro52 & & & & & & & & & & 2 \\
\hline Single positive & 22 & 12 & 4 & 0 & 0 & 0 & 4 & 1 & 0 & 2 \\
\hline Total & 45 & 27 & 18 & 0 & 5 & 8 & 17 & 9 & 0 & 24 \\
\hline
\end{tabular}

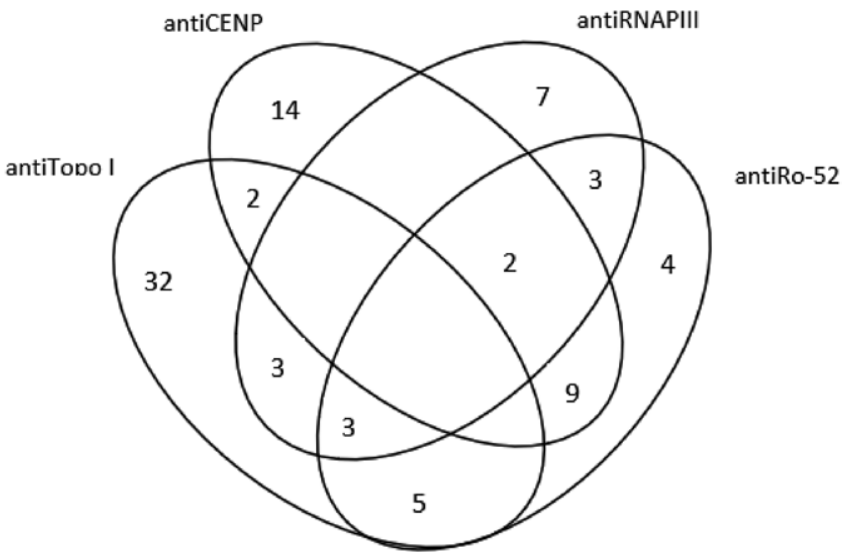

Figure 1. Diagram of disease-related antibodies against the four main autoantibodies [anti-centromere (antiCENP) anti-Topoisomerase I (antiTopo I), anti-RNA polymerase III (antiRNAP III) and anti-Ro52).

Conclusion: We presented the clinical and serologic features of the Turkish SSc patients from a new inception cohort. Clinical features of the SSc patients with single or multiple antibody positivity were not different.

References: None

Disclosure of Interests: None declared

DOI: 10.1136/annrheumdis-2020-eular.5030

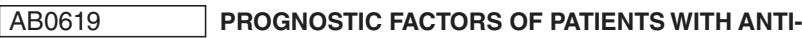 MDA5 ANTIBODY-POSITIVE DERMATOMYOSITIS COMPLICATED WITH INTERSTITIAL PNEUMONIA -A JAPANESE SINGLE CENTER STUDY-}

A. Umeda ${ }^{1}$, M. Sawada ${ }^{1}$, N. Watanabe ${ }^{1}$, M. Suzuki $^{1}$, T. Naganawa ${ }^{1}$, K. Ashihara ${ }^{1}$, M. Kurumizawa ${ }^{1}$, D. Hirano ${ }^{1}$, T. Hashimoto $^{1}$, J. Nishino ${ }^{1}$, S. Fukaya ${ }^{1}$, S. Yoshida ${ }^{1}$, H. Yasuoka ${ }^{1}{ }^{1}$ Fujita Health University, School of Medicine, Toyoake, Japan

Background: Anti-melanoma differentiation-associated gene 5 antibody (antiMDA5 Ab)-positive dermatomyositis (DM) is frequently associated with rapidly progressive interstitial pneumonia (RPIP), whose prognosis is assumed to be poor[1]. Although outcome of DM-RPIP has been reported to be improved by early immunosuppressive therapy, we still experience the cases with severe outcome. Only several reports mentioned the prognostic factors and they have not been fully elucidated.

Objectives: To identify the predictors of prognosis in patients with anti-MDA5 Ab-positive DM associated with interstitial pneumonia (DM-IP)

Methods: Anti-MDA5 Ab-positive DM-IP patients admitted to Fujita Health University Hospital between January 2010 and October 2019 were consecutively included and stratified into 2 groups, the survived and the deceased groups. DM was diagnosed according to the criteria proposed by Bohan and Peter[2]. Clinically amyopathic DM was diagnosed according to the criteria proposed by Sontheimer [3]. Diagnosis of IP was based on findings of high resolution CT scan (HRCT). The definition of RPIP was rapid exacerbation of hypoxemia or HRCT findings in a period of days to one month after the onset. Clinical features and prognosis of the patients were collected retrospectively and compared between groups. Candidates of predictors are extracted by the univariable analysis using Fisher's exact test for dichotic parameters and Wilcoxon signed-rank test for continuous parameters and multivariable analysis using logistic regression analysis. Receiver operating characteristic (ROC) curve analysis was examined to obtain the cut-off level. Survival was examined using Kaplan-Meier method and Log-rank test.

Results: Twenty-one patients were involved. Eight were deceased and 13 were survived. The deceased group had a higher ratio of male $(75 \%$ versus $25 \%, p=$ 0.018). All deceased cases were with RPIP and $67 \%$ in the survived cases. Levels of serum ferritin (4490 versus $646 \mathrm{ng} / \mathrm{mL}, \mathrm{p}=0.0026$ ), CRP (2.1 versus $0.9 \mathrm{mg} /$ $\mathrm{dL}, \mathrm{p}=0.0490)$, CK (1150 versus $290 \mathrm{U} / \mathrm{L}, \mathrm{p}=0.017$ ), AST (194 versus $108 \mathrm{U} / \mathrm{L}$ $p=0.025)$ and $\mathrm{LDH}(674$ versus $368 \mathrm{U} / \mathrm{L}, \mathrm{p}=0.011)$ were higher in the deceased group. Interestingly, skin ulcers were tended to be more frequent $(12.5 \%$ versus $87.5 \%, p=0.0587$ ), and anti-SS-A antibody was also more frequently detected (14.3\% versus $85.7 \%, p=0.0072$ ) in the survived group. Using ROC analysis cutoff values were $963 \mathrm{ng} / \mathrm{mL}$ for serum ferritin level (sensitivity $100 \%$, specificity $83 \%$ ), $0.7 \mathrm{mg} / \mathrm{dL}$ for CRP (sensitivity $75 \%$, specificity $69 \%$ ), $308 \mathrm{U} / \mathrm{L}$ for CK (sensitivity $88 \%$, specificity $77 \%$ ), $62 \mathrm{U} / \mathrm{L}$ for ALT (sensitivity $100 \%$, specificity $62 \%$ ), and $454 \mathrm{U} / \mathrm{L}$ for LDH (sensitivity $88 \%$, specificity $77 \%$ ). Patients were divided into two groups based on these cut-offs or based on dichotic parameters and surviva was examined between 2 groups. Except CRP and anti-SS-A antibody, survival was significantly worse in parameter-positive or higher groups. Interestingly, antiSS-A antibody-positive group had better outcome compared with those without. Conclusion: In our analysis, novel candidates such as serum CK, AST, and LDH levels were newly extracted and parameters previously reported was also included and those were also associated with the clinical outcome. In addition anti-SS-A antibody was identified as a novel protective factor associated with a good outcome.

References:

[1] Nakashima R, Hosono Y, Mimori T. Clinical significance and new detection system of autoantibodies in myositis with interstitial lung disease. Lupus 2016;25:925-33.

[2] Bohan A, Peter JB. Polymyositis and dermatomyositis. N Eng J Med 1975;292:344-7.

[3] Sontheimer RD. Dermatomyositis: an overview of recent progress with emphasis on dermatologic aspects. Dermatol Clin 2000;20:387-408.

Disclosure of Interests: None declared

DOI: 10.1136/annrheumdis-2020-eular.2708

\section{AB0620 \\ EFFECTIVENESS OF RITUXIMAB IN PATIENTS WITH EARLY DIFFUSE CUTANEOUS SYSTEMIC SCLEROSIS. A MULTICENTER ANALYSIS.}

I. Vázquez-Gómez ${ }^{1}$, J. Narváez ${ }^{2}$, J. Lluch Pons $^{2}$, M. Aguilar-Zamora ${ }^{3}$

L. Montolio-Chiva ${ }^{1}$, A. V. Orenes Vera ${ }^{1}$, E. Flores ${ }^{1}$, E. Valls-Pascual ${ }^{1}$,

D. Ybañez ${ }^{1}$, À. Martínez-Ferrer ${ }^{1}$, A. Sendra-García ${ }^{4}$, I. Torner Hernández ${ }^{1}$,

V. Núñez-Monje ${ }^{1}$, J. J. Alegre-Sancho' . 'Servicio de Reumatología, Hospital Universitario Dr. Peset, Valencia, Spain; ${ }^{2}$ Servicio de Reumatología, Hospital Universitari de Bellvitge, Barcelona, Spain; ${ }^{3}$ Servicio de Reumatolog ía, Hospital de Sagunto, Valencia, Spain; ${ }^{4}$ Fundación para el Fomento de la Investigación Sanitaria y Biomédica de la Comunidad Valenciana (FISABIO), Servicio de Reumatología y Farmacia, Hospital Universitario Dr. Peset, Valencia, Spain

Background: Rituximab (RTX) is effective in improving skin affection in patients with diffuse cutaneous systemic sclerosis (DcSSc). However, there are few data on early use of this drug.

Objectives: To evaluate RTX effectiveness for skin disease in patients with DcSSc of less than 3 years of evolution.

Methods: Multicenter, observational and retrospective study. Patients with DcSSc starting RTX within 3 years since first non-Raynaud symptom were recruited. Demographic variables, time of disease duration at the beginning of RTX, immune pattern and time on RTX treatment were collected. Effectiveness was defined as modified Rodnan skin score (mRSS) improvement. Evaluations were done by the same experienced rheumatologist. Patients subjective perception of skin hardening and/or tightness was evaluated. mRSS changes from baseline to 6 and 12 months after RTX beginning and, later on, to the last available observation were analysed using Wilcoxon test. Statistical analysis was performed with SPSS 20.0.

Results: 11 patients ( 8 women) were recruited from 2 university hospitals. Median age was 48 years (IQR 22). Median time since diagnosis to RTX beginning was 12 months (IQR 8). 5, 3 and 2 patients presented ATA +, RNPIII + and Ro-52 +, respectively. Median duration of RTX treatment was 12 months (IQR 68). Median baseline mRSS was 15.5 (IQR 18). Median mRSS after 6 and 12 months of RTX treatment and at last available mRSS evaluation was 15 (IQR 13), 14.5 (IQR 13) and 11 (IQR 16), respectively. mRSS showed statistically significant improvement at $6(29 \%, I Q R 37)$ and 12 month of RTX treatment (35\%, IQR 34) and, thereafter, at last available observation (39\%, IQR 51), compared to basal mRSS. Most patients reported subjective improvement at 6 (9 of 10 